\title{
Bilanz einer Bischöflichen Pressestelle
}

\author{
von Günter Graf
}

Am 21. Juli 1951 erschien in Münster die erste Ausgabe eines Pressedienstes der Diözese Münster unter dem Titel „ndm Nachrichtendienst Münster“. Bischof Dr. Michael Keller wies seinem Initiator und Herausgeber, dem damaligen Domvikar Heinrich Tenhumberg, die Aufgabe zu, auf diesem Wege einmal wöchentlich den im Bistum verbreiteten Zeitungen sowie dem Rundfunk Nachrichten, Berichte, Kommentare und Terminhinweise übermitteln zu lassen. Die redaktionelle Arbeit leisteten zunächst zwei Studenten. 1952 wurde der Verfasser dieses Berichts nach Abschluß des Studiums der Publizistik sowie nach redaktioneller Tätigkeit an zwei Tageszeitungen und einer Wochenzeitung verantwortlicher Redakteur.

Mit der Herausgabe dieses Pressedienstes war die Einrichtung einer Bischöflichen Pressestelle verbunden, der ersten in Deutschland und ab 1952 der ersten überhaupt, die von einem hauptamtlich tätigen Journalisten geleitet wurde. Während sie bis 1964 der Leitung des Seelsorgeamtes im Bischöflichen Generalvikariat unterstellt war, ist sie seither dem Bischof unmittelbar zugeordnet. Ihre personelle Besetzung wurde um einen zweiten Journalisten, der in der Pressestelle ausgebildet wurde, erweitert. Herausgeber des Pressedienstes jst seit 1966 das Bischöfliche Generalvikariat.

\section{Aufgaben}

1. Einmal wöchentlich, in der Regel an jedem Mittwoch, erstellt und verbreitet die Bischöfliche Pressestelle den „ndm Nachrichtendienst Münster". Auf durchschnittlich 6 bis 8 hektographierten Schreibmaschinenseiten informiert er in möglichst knapper, zeitungsgerecht redigierter Form über die Geschehnisse in der Diözese, die öffentliches Interesse erwarten lassen. Mit einer Auflage von 210 erreicht er ca. 50 Redaktionen der im Bereich des Bistums Münster und angrenzend verbreiteten Tageszeitungen (sowohl die Hauptredaktionen der Kopfblätter als auch deren Bezirks- bzw. Lokalredaktionen, ferner einige Redaktionen katholischer Tageszeitungen in den benachbarten Niederlanden), einen Teil der überregionalen katholischen Wochenzeitungen und Zeitschriften, den Westdeutschen Rundfunk, die Bezirksredaktionen der Katholischen Nachrichtenagentur (KNA), die Pressestellen der deutschen Diözesen, die Pressestellen von Behörden und anderen Einrichtungen des öffentlichen Lebens, die Referate, Führungs- bzw. Koordinierungsstellen im Bistum sowie interessierte Einzelbezieher, darunter die im Bistum wohnenden katholischen Landtags- und Bundestagsabgeordneten. Aktuelle Meldungen, die eine umgehende Veröffentlichung erfordern, werden unabhängig vom Erscheinungstermin des Pressedienstes telefonisch oder brieflich verbreitet.

Eine der Auflage bzw. dem Interesse entsprechende Monatspauschale berechtigt die Zeitungen und den WDR zur beliebigen Verwendung des durch den Pressedienst über-

Dr. Günter Graf, Vorstandsmitglied der Gesellschaft Katholischer Publizisten Deutschlands, ist Leiter der Bischöflichen Pressestelle Münster und Chefredakteur des "Nachrichtendienstes Münster" (ndm) sowie des "Zeitschriftendienstes - Mitteilungen der Arbeitsstelle für Zeitschriftenberatung". 
mittelten Materials. Das Abonnement, das nur der Information dient und nicht zum Abdruck berechtigt, wird mit monatlich 3,- DM berechnet.

Die im Pressedienst ausgewerteten Informationen werden entweder angeboten (im wesentlichen von den Referaten des Seelsorgeamtes, den Diözesanstellen, den katholischen Verbänden, Organisationen und Bildungseinrichtungen, den Bezirksassistenten im Bistum sowie von freien Mitarbeitern) oder eingeholt (durch Anfragen bei den genannten Stellen, Reportagen, Berichte über Veranstaltungen, durch Interviews u. a.). Die Auswahl und redaktionelle Bearbeitung erfolgen ausschließlich und in eigener Verantwortung der Bischöflichen Pressestelle. Eine Zensur findet nicht statt. Die Beiträge von Mitarbeitern werden honoriert (ca. 30,- DM für eine Schreibmaschinenseite; untere Grenze: 5,- DM pro Meldung).

Das Verhältnis der angebotenen zu den eingeholten Informationen hat sich in den vergangenen Jahren deutlich zugunsten des Angebots verlagert. Während die Pressestelle in der ersten Zeit fast ausschließlich auf ihre eigene Initiative angewiesen war, wobei z. T. beträchtliche Vorbehalte kirchlicher Stellen zu überwinden waren, beträgt die Relation heute etwa 70:30. Hierbei kommt dem Offentlichkeitsauftrag der Pressestelle ein gesundes Konkurrenzdenken der an der Publizität ihrer Arbeit interessierten kirchlichen Stellen zugute. Als nützlich hat sich das Prinzip erwiesen, alle von der Pressestelle herausgegebenen Informationen in den wöchentlichen Pressedienst aufzunehmen. Hierfür gilt sowohl der Gesichtspunkt der Repräsentation (des Veranstalters, über den berichtet wurde, bzw. des Informationsgebers) als auch - vor allem - der der Dokumentation.

Aus naheliegenden Gründen ist die ständige direkte Verbindung zum Bischof besonders wichtig. Der Leiter der Pressestelle nimmt an wöchentlichen Informationsgesprächen, die der Bischof mit seinen engsten Mitarbeitern führt, teil und erhält Einblick in den Terminkalender des Bischofs.

2. Seit der Gründung der Katholischen Nachrichtenagentur am 11. Januar 1953 erscheint der Pressedienst des Bistums Münster „in Verbindung mit KNA“; d.h. im wesentlichen, daß die Pressestelle neben ihrer eigenständigen Aufgabe die Funktion einer Bezirksredaktion der KNA wahrnimmt. Alle Meldungen von überregionaler Bedeutung werden telefonisch oder brieflich nach Bonn übermittelt. Dort werden sie entweder für den KNA-Pressedienst, den Westdeutschen Dienst, die Katholische Korrespondenz, den Dienst „Konzil - Kirche - Welt “ oder für den Informationsdienst ausgewertet. Gegenüber anderen Agenturen gilt der grundsätzliche Vorrang für KNA. Die auf diese Weise geregelte Zusammenarbeit mit der KNA kam erst nach Überwindung nicht unerheblicher Schwierigkeiten zustande. Die Gründer der KNA legten zunächst Wert darauf, daß der "Nachrichtendienst Münster" in die Bonner Zentralredaktion eingebracht werden sollte; der Leiter der Bischöflichen Pressestelle sollte in einen KNA-Bezirksredakteur "umfunktioniert" werden. Demgegenüber haben sich die Aufrechterhaltung und der Ausbau einer eigenständigen Bischöflichen Pressestelle sowie die Herausgabe eines von der. KNA unabhängigen Pressedienstes als richtig erwiesen. Hierfür spricht nicht zuletzt die Auswertung dieses Dienstes durch die an einer ausführlicheren Berichterstattung interessierten Hauptredaktionen der Tageszeitungen sowie durch deren - von der KNA nicht erreichten - Lokalredaktionen.

3. Als Kontaktstelle zwischen den Redaktionen der Zeitungen und des Rundfunks und dem Bischöflichen Generalvikariat bzw. dem Seelsorgeamt nimmt die Pressestelle eine weitere wichtige Funktion wahr. Unverzichtbare Voraussetzung ist die gute persönliche Verbindung des Leiters der Pressestelle zu den Journalisten in den Redaktionen. Dabei 
kommt ihm zustatten, daß er nicht in erster Linie als kirchlicher „Amtsträger" gesehen, sondern als journalistischer Kollege akzeptiert wird.

Anfragen, die von den Redaktionen an kirchliche Stellen oder Personen gerichtet werden, erreichen grundsätzlich zunächst die Pressestelle bzw. werden an diese verwiesen. Umgekehrt werden zur Veröffentlichung bestimmte Informationen kirchlicher Stellen durch die Pressestelle vermittelt. Das gleiche gilt für Pressekonferenzen, die von ihr organisiert werden und zu denen sie einlädt. Die Pressestelle selbst veranstaltet solche Konferenzen ein- bis zweimal jährlich mit dem Bischof oder dem Generalvikar. Anlässe bieten die Veröffentlichung des Haushaltsplans der Diözese, die Gründung bzw. Neustrukturierung kirchlicher Einrichtungen und andere Ereignisse von besonderem öffentlichem Interesse. (Beispiele aus der Vergangenheit: der Arbeitsbeginn kirchlicher Bildungseinrichtungen, die Wahl der Pfarrkomitees, die Gründung des Diözesankomitees, die Teilnahme des Bischofs am Konzil. 1955 wurde übrigens zum erstenmal in der Geschichte der Kirche der Haushaltsplan einer Diözese im Rahmen einer Pressekonferenz in Münster der Offentlichkeit vorgestellt.) Kirchliche Einrichtungen außerhalb der Stadt Münster, die der Bildung, der Caritas oder der Sozialarbeit dienen, waren das Ziel von Pressefahrten, die ebenfalls von der Pressestelle oder in Verbindung mit ihr veranstaltet wurden. Zugenommen hat in letzter Zeit die Zahl der Interviews, die Hörfunk und Fernsehen gegeben oder die durch die Pressestelle vermittelt wurden.

Einen besonderen Akzent erhält die kirchliche Offentlichkeitsarbeit durch die Vermittlung von Informationsgesprächen, die zwischen dem Bischof oder einem Dezernatsleiter und persönlich eingeladenen, verantwortlich tätigen Journalisten geführt werden. Ihr Ziel ist nicht eine unmittelbare Veröffentlichung, sondern die Darstellung von Zusammenhängen und Hintergründen bestimmter Sachverhalte.

4. Der Information der Offentlichkeit entspricht die Information "nach innen" mit gleichem Gewicht. Kirchliche Stellen und Personen (mit dem Bischof an erster Stelle) sind überfordert, wenn sie sich der Offentlichkeit gegenüber sachgerecht und informationsbereit verhalten sollen, ohne selbst hinreichend informiert zu sein. Die tägliche Arbeit der Pressestelle in Münster beginnt mit einer Auswertung von insgesamt ca. 35 Zeitungen und Zeitschriften. Wichtige Veröffentlichungen werden ausgeschnitten, mit Quellenangaben versehen, systematisch geordnet und in übersichtlicher Zusammenstellung dem Bischof, dem Generalvikar, den Weihbischöfen und den Dezernatsleitern auf möglichst schnellem Wege übermittelt. Dabei beanspruchen Berichte und Kommentare überregionaler oder ausländischer Zeitungen und Zeitschriften das gleiche Interesse wie Veröffentlichungen in den im Bistum erscheinenden Lokalausgaben der Tageszeitungen. Uber örtliche kirchliche Initiativen, die beispielhaft sein können, wird der Bischof vielfach ausschließlich auf diesem Wege informiert. Darüber hinaus werden Veröffentlichungen, die die Aufmerksamkeit einzelner Institutionen, Organisationen oder Personen erwarten lassen, diesen direkt zugestellt.

5. Vor allem in den ersten Jahren ihrer Tätigkeit hat die Bischöfliche Pressestelle Wert darauf gelegt, über ihre zentrale Funktion für die Diözese hinaus die kirchliche Pressearbeit innerhalb der lokalen und regionalen Strukturen der Diözese zu aktivieren. Hierzu boten sich in erster Linie die katholischen Verbände und Organisationen an. Eine systematische Schulung örtlicher Pressebeauftragter führte die Deutsche Kolpingsfamilie zusammen mit der Pressestelle durch. Weitere Kurse wurden für Mitglieder der Jugendorganisationen, der KAB, der CAJ und der Landjugend veranstaltet. Nach dieser Starthilfe sind die meisten Verbände dazu übergegangen, diese - für die öffentliche Darstellung ihres Selbstverständnisses und ihrer Aktivität in den Lokal- 
ausgaben der Tageszeitungen besonders wichtige - Informations- und Schulungstätigkeit mit eigenen oder ad hoc verpflichteten Fachkräften fortzusetzen. Nach wie vor bietet die Bischöfliche Pressestelle Themen aus dem Bereich der Massenmedien für Vorträge und Diskussionen im Rahmen der jährlichen Bildungsprogramme der Verbände an. Hierbei erweist es sich als Vorteil, daß der Leiter der Pressestelle zugleich für das Referat Film, Funk und Fernsehen in der Diözese Münster verantwortlich ist. Im regionalen Bereich nehmen die dem Bischöflichen Offizial für Oldenburg, dem Bischöflichen Kommissar für den Niederrhein, dem Stadtdechanten in Münster sowie den vier Bezirksdechanten im westfälischen Teil des Bistums zugeordneten Bezirksassistenten Funktionen der Offentlichkeitsarbeit wahr.

6. Redaktionelle Arbeit leistet die Bischöfliche Pressestelle neben der Herausgabe des ndm aufgrund besonderer Abmachungen mit dem Generalvikariat bzw. mit anderen Herausgebern durch die Redaktion der Zeitschriften „Unsere Seelsorge“ (Pastoralzeitschrift für das Bistum Münster, zweimonatlich), „Das Familienheim“ (Organ der Katholischen Familienheimbewegung in der BRD, monatlich), „forum “ (Organ des Presbyteriums im Bistum Münster, monatlich), "Zeitschriftendienst" (Mitteilungen der Arbeitsstelle für Zeitschriftenberatung, halbjährlich) sowie der in acht Diözesen verbreiteten „Schriftenreihe des Päpstlichen Werkes für geistliche Berufe “ (halbjährlich), der früheren Zeitschrift „Die große Ernte“.

\section{Erfabrungen}

\section{Nach außen}

Der Kontakt mit den Redaktionen setzt eine „mediengerechte ${ }^{\text {“ }}$ Arbeitsweise der Pressestelle voraus. Damit sind - abgesehen von der Redaktionsarbeit, die auf die Bedürfnisse und Gepflogenheiten von Presse und Rundfunk abgestimmt sein muß - auch psychologische und technische Gesichtspunkte angesprochen. Der Umgang mit Journalisten kennt eigene Gesetze, deren Ignorierung auf die Dauer mit einer Frustrierung noch so intensiver Bemühungen bestraft wird. Der Wert persönlicher Gespräche außerhalb des Dienstes - also in der Regel nach dem abendlichen Redaktionsschluß der Zeitungen - kann nicht hoch genug veranschlagt werden. Für die Arbeitszeit der Pressestelle gelten andere Regeln als die eines reglementierten Bürobetriebs; vor allem zwischen 17 und 19 Uhr muß die Pressestelle unmittelbar erreichbar sein. Unbedingt notwendig ist ein eigener Telefonanschluß, der von der Telefonzentrale des Generalvikariats und damit von dessen Dienstzeiten unabhängig ist.

Die praktischen Erfahrungen hinsichtlich des Abdrucks der durch den ndm verbreiteten Meldungen, Berichte und Kommentare veranlaßten die Pressestelle, das Schwergewicht auf knapp formulierte Meldungen zu legen, deren Überschriften nicht unbedingt auf einen „kirchlichen“ Inhalt schließen lassen. Es zeigt sich immer wieder, daß die Annahmebereitschaft in den Redaktionen der kirchlich nicht gebundenen Zeitungen hierdurch gefördert wird. Die belieferten Zeitungen und Zeitschriften veröffentlichen aus dem ndm-Angebot insgesamt durchschnittlich 5000 Druckzeilen im Monat. Diese relativ hohe Ziffer wird nicht zuletzt dadurch erreicht, daß zahlreiche Lokalredaktionen jeweils einige Tage nach dem Erscheinen des ndm solche Meldungen übernehmen, die von den Hauptredaktionen ihrer Zeitungen nicht ausgewertet wurden. Die Zahl der ndm-Beiträge, die von Hörfunk und Fernsehen sowie von den Nachrichtenagenturen dpa und AP übernommen werden, wurde nicht präzise ermittelt; sie ist ebenfalls beträchtlich. 
An der Spitze der Auswertung liegen Personalnachrichten; sie werden fast sämtlich und von allen Zeitungen gedruckt. Das gleiche gilt für Erklärungen des Bischofs und für amtliche, eine breite Offentlichkeit interessierende Verlautbarungen des Generalvikariats. Es ist schwierig, aus dem Abdruck der Meldungen und Berichte, die darüberhinaus verbreitet werden, einen Erfolgsschlüssel abzuleiten. Fest steht, daß innerbetriebliche, technisch bedingte Umstände in den Zeitungsredaktionen oft den Ausschlag geben. Uber Abdruck oder Nichtabdruck entscheidet z. B. nicht selten der Dienstplan (welcher Redakteur ist für die Auswahl jeweils verantwortlich?) oder nur die Tatsache, daß die Länge eines - irgendeines! - Berichts beim Umbruch zufällig auf einen freien Platz zugeschnitten ist. Es ist selbstverständlich, daß die Aufnahmebereitschaft in der "Sauregurkenzeit" (während der Sommerferien) besonders günstig ist; die Bischöfliche Pressestelle war immer gut beraten, wenn sie ihr Nachrichtenangebot darauf abstimmte. Dagegen sind die Zeitungsausgaben, die zu hohen kirchlichen Feiertagen erscheinen, in der Regel durch eine psychologische und eine technische Sperre blockiert: Das „kirchliche Soll“ wird hier durch redaktionelle Eigenleistungen erfüllt; zudem bedingt der Druck umfangreicherer Ausgaben (für jeweils zwei Tage) einen früheren Redaktionsschluß und eine entsprechende Reduzierung des aktuellen Teils.

Gute Erfahrungen wurden mit der gezielten, in einem persönlichen Begleitschreiben kommentierten Versendung bestimmter Meldungen gemacht. Auch in überregionale Tages- und Wochenzeitungen fanden auf diese Weise Veröffentlichungen der Bischöflichen Pressestelle Eingang.

In Einzelfällen haben sich regionale Pressekonferenzen - in Verbindung mit einem Sachreferat des Generalvikariats - als sinnvoll erwiesen. Beispiel: Als 1968 in Nordrhein-Westfalen die Volksschulen in Grund- und Hauptschulen umgewandelt und die konfessionellen Strukturen des Schulsystems einschneidend verändert wurden, führte die Pressestelle zusammen mit dem Schuldezernat des Bistums in fünf Städten Pressekonferenzen durch. Auf ihnen wurden die neue kirchliche Schulkonzeption dargelegt, Gedanken mit den Journalisten ausgetauscht und Hintergrundinformationen vermittelt. Der Wert dieser dezentralisierten Offentlichkeitsarbeit lag vor allem darin, daß in den Gesprächen mit den Journalisten und zusätzlich eingeladenen Schulfachleuten des jeweiligen Bezirks örtliche Sonderfälle angemessen berücksichtigt werden konnten - eine Aufgabe, die im Rahmen einer zentralen Pressekonferenz nicht hätte gelöst werden können.

Eine für das Selbstverständnis und die Effektivität kirchlicher Offentlichkeitsarbeit entscheidende Voraussetzung resultiert aus der Erfahrung, daß Presse und Rundfunk sich in der Regel sachlich korrekt und loyal verhalten, wenn sie ins Vertrauen gezogen werden. Ein besonders instruktives Beispiel sei hier wegen seiner symptomatischen Bedeutung zitiert:

Als ein Pfarrer einer angesehenen Großstadtgemeinde wegen Schwierigkeiten, die sich in der Verwaltung der Gemeinde ergeben hatten, sein Amt im Einvernehmen mit dem Generalvikariat niederlegte, wurden Presse und Rundfunk zwei Tage vor der Demissionierung durch die Bischöfliche Pressestelle über diesen Vorgang sowie über seine Hintergründe informiert. Das Ergebnis war eine sachliche Berichterstattung mit einer Zitierung der Gründe, die der Pfarrer für seine Amtsniederlegung der Gemeinde mitgeteilt hatte. Hätte man versucht, diesen „Fall“ durch unvollständige Information zu bagatellisieren oder gar totzuschweigen, wäre er erfahrungsgemäß durch journalistische Recherchen einseitig dargestellt worden. Durch die rechtzeitige offene Darlegung des Sachverhalts und seiner Zusammenhänge gab es keinen Grund für Spekulationen. 


\section{Nach innen}

Dieses Beispiel akzentuiert zugleich eine wesentliche Einsicht in die „innerbetriebliche“ Standortbestimmung der Bischöflichen Pressestelle. Ein Pressereferent, dessen Aufgabe sich in der Hauptsache darin erschöpft, amtliche Verlautbarungen zu multiplizieren, ist fehlinvestiert. Das gilt für ein staatliches oder wirtschaftliches Unternehmen in gleichem Maße wie für eine Diözese. Seine - selbstverständliche - kirchliche Gesinnung, nicht zuletzt seine Loyalität vor allem gegenüber dem Bischof, darf ihn nicht zu einer unkritischen Treue verleiten, die ihn leicht in die Gefahr bringt, seine Tätigkeit auf die Funktion eines Briefträgers zu verkürzen.

Die Bereitschaft kirchlicher Amtsträger, ihre Pressestelle nicht nur als unbequemes Zugeständnis an die Vorstellung von einer "modernen “ Kirche zu tolerieren, sondern deren Arbeit als unverzichtbaren Beitrag zur Präsenz der Kirche in der Welt und als Teil ihres Dienstes zu akzeptieren und zu fördern, ist erste Voraussetzung für die Wirksamkeit einer Bischöflichen Pressestelle. Dazu gehört auch die Einsicht, daß kritische Haltungen in der Kirche und "Ärgernisse“ aus der öffentlichen Selbstdarstellung nicht ausgeklammert werden dürfen; sie trägt zugleich der Eigengesetzlichkeit der öffentlichen Kommunikation in unserer Gesellschaft Rechnung. Daß ihre Ignorierung beträchtlichen Schaden für die Kirche zur Folge haben kann, hat sich am „Fall Billerbed" erwiesen: Die Tatsache, daß in diesem Fall eines katholischen Arztes, der bei seiner Bewerbung um das Belegrecht an einem katholischen Krankenhaus seine Ehescheidung und Wiederverheiratung verschwiegen hatte und daraufhin entlassen wurde, die Pressestelle nicht hinreichend informiert war und formaljuristische E.rwägungen gegenüber publizistischen den Vorrang bekamen, führte zu einer publizistischen Eskalation mit der Kirche als Zielscheibe massiver Attacken.

Somit kann die Bischöfliche Pressestelle ihrer Aufgabe nur dann gerecht werden, wenn sie über innerkirchliche - auch „delikate ${ }^{\text {“ }}$ - Vorgänge laufend informiert und ihre Verantwortung für ihre publizistische Arbeit durch fachliche Beratung und entsprechende Entscheidung qualifiziert wird. Nur unter dieser Voraussetzung kann sie die Offentlichkeit sachgerecht über die kirchliche Wirklichkeit informieren. Daß sie über eine angemessene personelle, finanzielle und technische Ausrüstung verfügen muß, versteht sich von selbst - ebenso wie ihre fachliche journalistische und „kirchliche“ Qualifikation, die den Erwartungen an ihren Auftraggeber entsprechen muß.

\section{SUMMARY}

It began as a regional news service for the diocese of Münster (Germany) started on July 21, 1951, and became in the following months the Diocesan Information Office. After 18 years of work the director of the office-a trained (lay) journalist-describes here his experiences. Duties of the office are: editing a weekly news service, cooperation with the German Catholic News Agency (KNA, Bonn), contacts with press and radio editorial offices within the limits of the diocese, information of the bishop and the diocesan government on current affairs, the promotion of Catholic press work in general. From the long years of experience it seems to be clear that only a professional journalist can handle these things. Beside that, a loyal relationship based on mutual confidence between bishop and press officer is a must for successful work. The information office has to inform the public, even about unpleasant events in the diocese, promptly and fully. 